\title{
LAS GUÍAS DIDÁCTICAS DE EDUCACIÓN SEXUAL, LA IMPUGNACIÓN RELIGIOSA Y LA REGULACIÓN DE LA NORMALIDAD SEXUAL EN COSTA RICA, 1985-1998
}

\author{
The sexual education teaching guides, the religious contest, and \\ the regulation of sexual normality in Costa Rica, 1985-1998
}

\section{José Daniel Jiménez Bolaños ${ }^{\alpha}$}

Fecha de recepción: 10/12/2020 • Fecha de aceptación: 08/03/2021

Resumen. Este artículo tiene por objetivo analizar en perspectiva histórica y a través de fuentes periodísticas, el desarrollo de la redacción, discusión e implementación de las guías didácticas de educación sexual en Costa Rica entre 1985-1998. Al momento de entrar en funcionamiento, fueron impugnadas por las principales autoridades religiosas del país. La impugnación religiosa motivó el despliegue de la opinión pública, generando voces a favor y en contra de su intervención en el desarrollo de las guías. El ir y venir entre los esfuerzos estatales y la negativa eclesiástica llevó a que las guías fueran reformuladas, cambiando aspectos puntuales en su contenido, y dándoles un enfoque menos secular y más cercano a los dogmas cristianos. Este proceso de polarización evidenció que, en el país, existían dos proyectos distintos en la construcción y regulación de la normalidad sexual en las aulas, cuyo punto de discordia fue la primacía o no de los ejes religiosos. Las guías tuvieron un efecto muy limitado a nivel educativo.

Palabras clave: Sexualidad; Educación; Educación sexual; Costa Rica; Historia.

Abstract. The objective of this article is to analyze in historical perspective and through journalistic sources, the development of the writing, discussion, and implementation of the sexual education teaching guides in Costa Rica since 1985. From their very inception they were contested by the country's

\footnotetext{
${ }^{\alpha}$ Escuela de Historia, Facultad de Ciencias Sociales, Universidad de Costa Rica, San Pedro de Montes de Oca 11501, San José, Costa Rica. josedaniel.jimenez@ucr.ac.cr (iD https://orcid.org/0000-0002-8868-7729
}

Cómo citar este artículo: Jiménez Bolaños, José Daniel. «Las guías didácticas de educación sexual, la impugnación religiosa y la regulación de la normalidad sexual en Costa Rica, 1985-1998». Historia y Memoria de la Educación 15 (2022): 451-478 
main religious authorities. This religious challenge gave rise to an outburst of public opinion, with voices for and against the clergy's intervention in the development of the teaching guides. The tug of war between state efforts and ecclesiastical refusal led to a reformulation of the guidelines, with changes in their content that conferred to them an approach that was less secular and closer to Christian dogmas. This process of polarization evidenced the two different projects that coexisted in the country in the construction and regulation of sexual normality in the classroom, the point of contention being the predominance otherwise of the religious axes. The guides had a limited effect at the educational level.

Keywords: Sexuality; Education; Sexual education; Costa Rica; History.

\section{INTRODUCCIÓN}

La discusión sobre la educación sexual tiene una larga historia en Costa Rica, en dichas discusiones se encuentran dos actores principales; el Estado y la Iglesia y, a lo largo de los años, los encuentros y desencuentros entre las posturas de ambas partes han provocado momentos de tensión, de cooperación y de directa oposición. Desde la reforma educativa de 1886 en la cual se dio un proceso de centralización, el Poder Ejecutivo se convirtió en el ente que dirigía y administraba la enseñanza en el país. En su afán de disminuir la influencia de los municipios y la Iglesia, se promovió la secularización ${ }^{1}$, eliminando el peso que tenían los contenidos religiosos en el currículum educativo. ${ }^{2}$ A partir de 1920 empiezan a surgir de forma fragmentaria en las memorias de la Secretaría de Instrucción Pública, menciones acerca de la importancia y necesidad de incluir contenidos vinculados con la higiene, el venerismo y la salud sexual en los planteles educativos. Al llegar la década de 1940, bajo la presidencia de Rafael Ángel Calderón Guardia (1940-1944), se da un nuevo acercamiento del Poder Ejecutivo con la Iglesia, unión que se materializa a través de la eliminación de las leyes liberales que habían limitado la influencia religiosa en el ámbito educativo desde finales del siglo XIX. ${ }^{3}$

\footnotetext{
${ }^{1}$ También se dieron reformas como la secularización de los cementerios, la expulsión del obispo y los jesuitas, la prohibición del establecimiento de órdenes monásticas, así como de la colecta de limosnas sin autorización previa y las procesiones con imágenes fuera de los templos.

${ }^{2}$ Iván Molina Jiménez, La educación en Costa Rica de la época colonial al presente. (San José: PEN-EDUPUC, 2016), 134-135.

${ }^{3}$ David Díaz, Reforma sin alianza, discursos transformados, interés electoral, triunfos dudosos. (San José: Editorial UCR, 2010), 15.
} 
En 1968 surge el Plan Nacional de Educación Sexual y Planificación Familiar, un proyecto que contó con apoyo estatal y una fuerte presencia del sector privado. Su objetivo era capacitar primero a los docentes, para luego llevar los contenidos a las aulas. En esa ocasión, la Iglesia no mostró oposición, incluso organismos como el Centro de Orientación Familiar y el Centro de Integración Familiar — ambas instituciones privadas de corte religioso- tuvieron un papel de gran importancia en el desarrollo e implementación del Programa. Al llegar la década de 1980, gran parte de este había quedado rezagado o en el olvido, por lo que surge nuevamente la necesidad de desarrollar algún tipo de proyecto para facilitar la educación sexual.

Este artículo tiene por objetivo analizar en perspectiva histórica y a través de fuentes periodísticas, el desarrollo de la redacción, discusión e implementación de las guías didácticas de educación sexual en Costa Rica entre 1985 y 1998. Se parte de la idea de la sexualidad como una construcción social, ${ }^{4}$ en donde la idea de la normalidad no se da automáticamente, sino que es el resultado de luchas de poder simbólico y material. ${ }^{5}$ En palabras del historiador Jonathan Katz, «se inventa en el discurso como algo que está afuera del discurso. Se fabrica en un discurso particular como algo que es universal. Se construye en un discurso históricamente específico como algo que es atemporal. Se construyó muy recientemente como algo que es muy antiguo». ${ }^{6}$ En ese sentido, analizar los discursos de los diferentes actores involucrados en la discusión de las guías didácticas, permite evidenciar los diferentes proyectos políticos de construcción de la normalidad sexual presentes en el ámbito público.

Tomando en cuenta que para el 2018 —en el contexto de las elecciones presidenciales - hubo grupos de padres y madres de familia que cerraron centros educativos de primaria en el país, como forma de protesta por la aplicación del Programa de Afectividad y Sexualidad Integral del Ministerio de Educación Pública (MEP), ${ }^{7}$ es claro que las disputas que

\footnotetext{
${ }^{4}$ Jeffrey Weeks, Sexuality. (London: Routledge, 2003); Michel Foucault, Historia de la sexualidad vol. 1: La Voluntad del saber. (Buenos Aires: Siglo XXI, 2003).

5 Óscar Guasch, La crisis de la heterosexualidad. (Barcelona: Laertes, 2000), 17-18.

${ }^{6}$ Jonathan Katz, La invención de la heterosexualidad. (México: Editorial Me cayó el veinte, 2012), 253.

7 María Peña Bonilla, «Afectividad y sexualidad integral: ¿la manzana de la discordia para el sistema educativo?» Universidad de Costa Rica. https://www.ucr.ac.cr/noticias/2018/03/25/afectividad-y-sexualidad-integral-la-manzana-de-la-discordia-para-el-sistema-educativo.html (consultado el 18 de enero, 2021).
} 
surgen a raíz de este tema siguen generando mucha polémica. Por lo tanto, se vuelve necesario un análisis histórico que permita visualizar el desarrollo, así como los cambios y permanencias, de esta temática.

\section{ORIGEN DE LAS INICIATIVAS PARA LA ELABORACIÓN DE LAS GUÍAS DIDÁCTICAS}

Durante la administración del presidente Luis Alberto Monge (19821986), el entonces ministro de Educación, el Lic. Eugenio Rodríguez, firmó un convenio con el Fondo de las Naciones Unidas para Actividades de Población (UNFPA), cuyo principal objetivo era introducir en el país un proyecto de educación sobre la natalidad y, en general, sobre cuestiones demográficas. ${ }^{8}$ Como parte de ese convenio, se empezaron a redactar unas guías de educación sexual para el tercer ciclo de enseñanza (séptimo, octavo y noveno). La elaboración estas contó con el auspicio del Programa Regional de Educación en Población de la Unesco, el cual había iniciado funciones en $1974 .{ }^{9}$ Esta iniciativa estaba enmarcada en un contexto de crisis de financiamiento de la educación pública en el país. ${ }^{10}$

Para inicios de 1985, se formó una comisión integrada por el profesor Efraín Valerio, quien era experto en biología, y el sacerdote Alfredo Madrigal. Esta comisión se dio a la tarea de redactar y preparar la primera versión durante el segundo semestre de 1986 y la primera mitad de 1987. Esta primera versión ilustrada fue enviada a técnicos, religiosos y especialistas para su revisión. Los aportes que surgieron fueron incorporados en la segunda versión, entre septiembre y noviembre de 1987, lo que permitió que durante el ciclo lectivo de 1988 fueran utilizadas de manera experimental en los liceos de San Marcos de Tarrazú, San Pablo de León Cortés, Santa María de Dota, Pavas y en el Colegio Superior de Señoritas. A finales de 1988, se preparó una tercera versión, y el 10 de noviembre de ese año, el obispo auxiliar de San José, Antonio Troyo, envió una carta al ministro de Educación de ese entonces, el Dr. Francisco Antonio Pacheco, con una serie de demandas acerca de la ausencia de aspectos morales y cristianos en los textos.

\footnotetext{
${ }^{8}$ Ronald Espinoza y Gabriela Quirós, «Camino tortuoso de unas guías», La Nación (San José), 25 noviembre de 1991, 5.

9 Emilia Mora Gamboa, «¿Por qué no en los colegios?», La Prensa Libre (San José), 5 febrero de 1992, 1.

${ }^{10}$ Molina, La educación en Costa Rica, 440-446.
} 
A raíz de esto, en 1989 se nombra una comisión integrada por cuatro delegados del MEP y cuatro de la Iglesia Católica, con el objetivo de plantear recomendaciones generales. En junio de 1990, el nuevo ministro de Educación, el Lic. Marvin Herrera, instaló una segunda comisión mixta para realizar algunos cambios, elaborando la versión final. No obstante, para marzo de 1991, el arzobispo de San José, Román Arrieta, pidió la suspensión de la entrega. ${ }^{11}$

Para julio de 1991, después de varios meses de espera, la Conferencia Episcopal de Costa Rica (CECOR), órgano que agrupa a los obispos del país, había terminado de leer y analizar los tres documentos, y en una carta enviada al ministro de Educación, venían especificadas tres sugerencias: la primera consistía en la elaboración de un marco doctrinal sobre aspectos de interés particular para la Iglesia, segundo, que los estudiantes de religión fueran incluidos y priorizados en la administración y uso, así como una adecuada capacitación a los profesores en general. ${ }^{12}$

En ese momento, el MEP aceptó las observaciones. El marco teórico doctrinal tenía la función de permitir que ciertos aspectos pudieran ser leídos en clave católica. Dicho marco teórico fue redactado y entregado a los obispos para su estudio, y sus autores fueron los presbíteros José Celada Moreno y Osvaldo Brenes Álvarez, los asesores nacionales de educación religiosa. En su momento, el arzobispo se mostró satisfecho con dicho documento y comentó que era lo que faltaba para complementar los documentos. ${ }^{13}$

\section{PRIMERAS REACCIONES AL PROYECTO DE LAS GUÍAS DIDÁCTICAS}

Cuando la Iglesia pidió retener el programa con el objetivo de revisarlo y asegurarse que no atentara contra su doctrina, ${ }^{14}$ fue una de las pocas veces donde en la prensa nacional se mencionó dicho proyecto

\footnotetext{
11 Ronald Espinoza y Gabriela Quirós, «Camino tortuoso de unas guías», 5.

12 Efraín Valerio Charpentier, «Los obispos deben cumplir compromiso con el MEP», La Prensa Libre (San José), 26 noviembre de 1991, 9.

13 Efraín Valerio Charpentier, «Los obispos deben cumplir compromiso con el MEP», 9.

${ }^{14}$ Lupita González, «Factores religiosos frenan educación sexual en el país», La República (San José), 6 junio de 1988, 8.
} 
que ya llevaba años en desarrollo. El vicario episcopal de la Pastoral Social, el padre Carlos Humberto Abarca, argumentaba que «no hay oposición en que se suministre a los jóvenes, e incluso a los niños, información sexual, el problema aquí es la forma de educarlos que se quiere implantar». ${ }^{15}$ Por su parte, Aquiles Ureña quien era el presidente del Movimiento Familiar Cristiano, afirmaba que «ahí no se tomaba en cuenta que el acto sexual es una relación de amor entre dos personas, sino que, se presentaba la relación como algo casual, algo sin importancia, donde lo único que interesa es el "hacerlo por hacerlo", y no el amor» ${ }^{16}$ Como forma de justificar dicha oposición, se confirmaba que la Iglesia trataba de prevenir el embarazo adolescente ${ }^{17}$ — visto como el problema número uno de la falta de educación sexual - mediante la preparación prebautismal y preconfirmal, así como con los cursos prematrimoniales.

Una de las críticas más tempranas vino de la mano de la educadora e integrante del Opus Dei, Helena Ospina, quien para 1987 establecía que los programas de educación sexual que se habían tratado de desarrollar en Costa Rica, no buscaban sus fundamentos en los criterios de la ley moral, natural, sino que la mayoría de las veces quedaban supeditados al «subjetivismo y relativismo de corrientes materialistas y de presiones económicas de financiación de políticas demográficas antinatalistas ${ }^{18}$ para luego dictaminar que las consecuencias de este tipo de educación, desarrolladas al margen de la moral eran palpables a la vista; «el aumento en embarazos en estudiantes de secundaria, el aborto, la masturbación, el homosexualismo, el bisexualismo, el lesbianismo».19 Además, manifestaba que, en el fondo, esta educación debería ser impartida por los padres de familia y que lo que estaba haciendo el Estado era una

\footnotetext{
15 Evelyn Ardón Rodríguez, «No hay oposición en que se imparta la educación sexual», La Prensa Libre (San José), 17 agosto de 1990, 4.

16 Evelyn Ardón Rodríguez, «No hay oposición en que se imparta la educación sexual», 4.

${ }_{17} \mathrm{Al}$ igual que en muchos lugares, el embarazo adolescente en Costa Rica no es un fenómeno homogéneo, ya que se encuentra vinculado con el nivel educativo y socioeconómico, así como las diferencias entre zonas rurales y urbanas, el acceso a servicios de planificación familiar y el machismo. Según una investigación, el número medio de hijos nacidos vivos de mujeres entre 15 y 19 años de edad pasó de 0,19 a 0,17 entre 1986 y 1993, ver: Hilda Núñez Rivas y Ana Rojas Chavarría, «Revisión conceptual y comportamiento del embarazo en la adolescencia en Costa Rica, con énfasis en comunidades urbanas pobres», Revista Costarricense de Salud Pública, vol. 17, n. 13 (1998), 1-4.

18 Helena Ospina, «Educación sexual integral», La Nación (San José), 28 diciembre de 1987, 16.

19 Helena Ospina, «Educación sexual integral», 16.
} 
imposición, suplantando el derecho irrenunciable de los padres. ${ }^{20}$ Finalmente arremetió contra la metodología participativa que se estaba proponiendo, aduciendo que dicha estrategia pedagógica era nefasta, «no puede menos que degenerar en estímulo de la curiosidad y experimentación sexual entre jóvenes [...] dictado por lo cambiante de las modas hedonistas y materialistas de nuestra sociedad de consumo». ${ }^{21}$

Llama la atención que, en dicha argumentación, una de las principales amenazas de una inapropiada educación sexual, es la posibilidad de que los y las estudiantes se vuelvan homosexuales o lesbianas. Se visualizaba a la educación sexual como un proyecto que idealmente fortalecería la heterosexualidad de la población más joven, frente a estas amenazas que acechaban alrededor. La crítica de Ospina muestra que, para finales de la década de 1980 e inicios de la de 1990, estaban entrando en colisión los diferentes proyectos políticos que buscaban construir y regular la normalidad sexual, cada uno de ellos en un esfuerzo por construir su discurso en la verdad. ${ }^{22}$ Por un lado, el gobierno y las autoridades educativas y, por otro, la sexualidad propuesta por la Iglesia y sus seguidores, para quienes la castidad, la abstinencia y el matrimonio eran las únicas formas de expresión sexual permitidas. Todo aquel proyecto o iniciativa política que no colocara al matrimonio en el centro, se volvía una amenaza y un enemigo a su visión de cómo debería construirse y regularse la normalidad. ${ }^{23}$

\footnotetext{
20 A nivel global, uno de los principales temas que generaba críticas hacia la educación sexual provenía directamente de los padres y madres de familia, quienes argumentaban sobre su derecho a educar a sus hijos e hijas de acuerdo con sus principios y valores particulares. Estas críticas empezaron a ganar fuerza a partir de las décadas de 1960 y 1970 en el marco de los cambios generados por la creación de la píldora anticonceptiva en 1968, los movimientos feministas y de liberación sexual, así como la expansión sin precedentes de la cultura de masas. Uno de los casos más notables se dio en Dinamarca en 1971, cuando un grupo de padres interpuso una queja ante la Corte Europea de Derechos Humanos, debido a que un año antes se había promulgado la obligatoriedad de impartir educación sexual de forma integrada a lo largo del currículo educativo en ese país, lo cual hacía más difícil retirar a los niños de dichas lecciones. Al enmarcar dicha educación como vulgar y contraria a su religión, los demandantes argumentaban que el Estado tenía que respetar el derecho de los padres de asegurar una educación en conformidad con sus propias convicciones religiosas y filosóficas. La Corte no les dio la razón, estableciendo que dicha educación no constituía un adoctrinamiento hacia algún tipo específico de comportamiento sexual. Por el contrario, en Estados Unidos durante la década de 1980, nueve estados decretaron específicamente que los padres podían retirar a sus hijos de las clases, en otros cinco estados se requería el consentimiento escrito de los padres antes de que los estudiantes pudieran recibir la materia, ver: Jonathan Zimmerman, A global history of sex education (New Jersey: Princeton University Press, 2015), 80-97.

${ }^{21}$ Helena Ospina, «Educación sexual integral», 16.

22 Michel Foucault, El orden del discurso (Barcelona: Tusquets, 1999).

${ }_{23}$ Mary Louise Adams, The Trouble with Normal: Postwar Youth and the Making of Heterosexuality (Toronto: University of Toronto Press, 2012).
} 


\section{LAS DECLARACIONES DEL PAPA}

Las guías didácticas que venían elaborándose desde 1985 estaban a la expectativa de ser publicadas y difundidas entre los docentes de colegios públicos. En medio de la espera a que la Iglesia diera su sentencia en torno a estas, el 19 de noviembre de 1991, en una audiencia en el Vaticano, el Papa Juan Pablo II recibió las cartas credenciales presentadas por el nuevo embajador de Costa Rica ante la Santa Sede, Manuel Hernández Gutiérrez. En dicha audiencia, el Papa abordó el tema de la educación sexual. Sus palabras desataron una polémica que involucró a múltiples sectores de la población y colocó al tema en el centro de la discusión pública. Entre otras cosas, el Papa manifestó que:

Un campo de particular importancia, tanto para la Iglesia como para las instituciones civiles, es la familia y la juventud. Por ello, los Pastores, en el ejercicio de su misión evangelizadora, no han dejado de manifestar su preocupación ante determinadas campañas antinatalistas y sobre ciertos programas escolares de educación sexual. En efecto, corresponde a la misión de la Iglesia formar las conciencias y ofrecer criterios en materias delicadas que inciden de modo tan relevante en el comportamiento y en los principios morales de las personas, sobre todo de los niños y los jóvenes. ${ }^{24}$

El mismo concepto usado en críticas anteriores vuelve a resurgir en estas declaraciones, el antinatalismo deviene un término que amalgamaba la oposición de la Iglesia y otros sectores de la población frente a la institucionalización de una educación sexual y un servicio de planificación familiar modernas en el país. ${ }^{25} \mathrm{Al}$ igual que con las críticas de la

24 «Del Papa Juan Pablo II al pueblo de Costa Rica», La Nación (San José), 21 noviembre de 1991, 18.

${ }^{25}$ Hacia la década de 1980, Estados Unidos bajo la presidencia de Ronald Reagan, anunció un giro radical, afirmando que la planificación familiar ya no era un tema prioritario en su agenda mundial, adoptando una posición «neutral» en torno al crecimiento de la población, además de una oposición categórica al aborto, ver: Warren Robinson \& John Ross, The global family planning revolution. 3 decades of population policies and programs. (Washington: The International Bank for Reconstruction and Development, 2007), 9. En Costa Rica, los servicios de planificación familiar contaban con apoyo estatal desde 1968, no obstante, durante la década de 1980 diferentes sectores sociales, impulsados por un ambiente de creciente conservadurismo sociocultural, autoproclamados «provida» y abajo la égida de la «defensa de la familia», empezaron a recurrir a los medios de comunicación para externar sus posiciones con relación a las políticas del Estado en materia demográfica, por ejemplo: Abelardo Rivera, «Una paradoja y sus variantes», La Nación (San José) 10 de febrero de 1987, 16; 
Iglesia anteriormente mencionadas, el Papa argumentó que, sin una dimensión ética, esta educación fácilmente podía convertirse en permisividad. ${ }^{26}$ Esta afirmación proveniente del Vaticano suscitó reacciones inmediatas.

Las maestras fueron de las primeras en reaccionar. En un artículo de La Prensa Libre, se entrevistó a varias docentes para conocer su opinión acerca de las declaraciones del Papa. Entre ellas, la directora de la Escuela España, María Concepción Ramírez, manifestó que tanta campaña de educación sexual no había funcionado en el país, ya que el número de embarazos adolescentes seguía subiendo, además que, si se establecía esta educación desde edades tempranas, se corría el peligro de dar «rienda suelta a sus impulsos. Estoy de acuerdo con el Papa. El sexo es algo natural pero nunca debe olvidarse la moral ligada a esta educación». ${ }^{27}$ Una maestra afirmaba que había recibido "algunos cursos de educación sexual y nunca me atreví a impartirlos en clases [...] para no inducir a los niños a hacer algo incorrecto» ${ }^{28}$ otra dijo que «sí es necesario impartirla, yo la doy desde primer grado, pero considero que debe servir para encauzar a los niños y darles valores que lleguen hasta los padres de familia», ${ }^{29}$ finalmente, una maestra expresó que «el Papa está en lo correcto, debe prevalecer la moral». ${ }^{30}$

Por su parte, el gobierno rechazó inicialmente las críticas del Papa, el presidente Rafael Ángel Calderón aseguraba que dichas declaraciones se debían a informaciones equivocadas. Por su parte, el ministro de Educación, Marvin Herrera, advirtió que las guías didácticas no iban a salir oficialmente hasta tener el visto bueno de la Iglesia. Asimismo, ante esta situación, el presidente decidió enviar con urgencia una nota

\footnotetext{
«Familia, contra concepción y moral», Eco Católico (San José), 3 de enero de 1988, 8; Eduardo Ramírez, "AID impone políticas de natalidad en el país», Universidad (San José), 31 de mayo de 1989, 21. Por lo tanto, en el momento en que el Papa hace sus declaraciones, la planificación familiar y las políticas demográficas eran ya temas recurrentes de debate por parte de la opinión pública.

26 «Papa critica a Costa Rica», La República (San José), 20 noviembre de 1991, 2.

27 Emilia Mora Gamboa, «Maestras apoyan al Papa», La Prensa Libre (San José), 20 noviembre de 1991, 3 .

28 Emilia Mora Gamboa, «Maestras apoyan al Papa», 3.

29 Emilia Mora Gamboa, «Maestras apoyan al Papa», 3.

30 Emilia Mora Gamboa, «Maestras apoyan al Papa», 3.
} 
aclaratoria al Vaticano. ${ }^{31}$ Los proyectos políticos en torno a la normalidad ya se encontraban en pugna, y en medio de esa batalla, el sector estatal y educativo empezó a dar concesiones al sector eclesiástico.

La Asamblea Legislativa también reaccionó rápidamente a las declaraciones del Papa. La diputada del Partido Unidad Social Cristiana (PUSC), Nury Vargas, calificó como una intromisión a dicho discurso, y censuró al Papa por su posición frente a las políticas de educación sexual en el país. La reacción de Vargas suscitó a su vez, rebotes de integrantes de su propio partido y del Partido Liberación Nacional (PLN), entre los argumentos que salieron a la luz en el Congreso, se criticó la labor de los gobiernos pasados — especialmente el de Óscar Arias- por ser los responsables de haber aprobado el proyecto, y varios confrontaron a Vargas, manifestando que las declaraciones del Papa no se podían interpretar como una intromisión ya que este «habla como guía espiritual». ${ }^{32}$

En defensa de las guías, Rolando Zamora, director del Centro Nacional de Didáctica, órgano responsable de su elaboración, declaró que, desgraciadamente, la educación sexual seguía siendo tabú, que estas no eran escandalosas, y que tenían un aspecto moral, el cual permitía que los estudiantes analizaran críticamente el comportamiento humano. ${ }^{33}$

El vicecanciller de la Santa Sede, Claudio Celli, aclaró que el Papa no había hecho ningún reproche al gobierno de Costa Rica, sino que nada más expresaba una preocupación, compartida con los obispos, de que no se ajustaran a los dogmas de la Iglesia. El obispo Jorge Aníbal Rojas incluso buscó desmentir al presidente, afirmando que el Papa no estaba «mal informado».34 Por su parte, el ministro de relaciones exteriores, Bernd Niehaus, aseguró que lo expuesto por el Papa no era una crítica directa, sino «su posición universal clara y general en materia de política de educación sexual». ${ }^{35}$ En esa misma sintonía, el arzobispo de San José, y presidente de la Conferencia Episcopal de Costa Rica (CECOR), Román

\footnotetext{
31 «Gobierno rechaza críticas del Papa», La República (San José), 21 noviembre de 1991, 2.

32 «Nury Vargas critica al Papa», La República (San José), 21 noviembre de 1991, 2.

33 «Vaticano dice que crítica no fue directa», La Nación (San José), 22 noviembre de 1991, 4.

34 «Vaticano dice que crítica no fue directa», 4.

35 «Vaticano desmiente críticas a Costa Rica», La República (San José), 22 noviembre de 1991, 4.
} 
Arrieta, aseveró que el Papa tenía pleno derecho de expresar sus preocupaciones y eso no significaba una injerencia en los asuntos internos del Estado. Para el arzobispo, la educación sexual era una necesidad urgente para la juventud, concluyendo que los obispos, al ser también ciudadanos, tenían derecho de opinar y expresar sus inquietudes. ${ }^{36}$

Para el ex ministro de Educación, Víctor Brenes Jiménez, el tema se había manejado hasta ahora de una forma secreta, en círculos demasiado estrechos y a espaldas de la opinión pública, por lo que las declaraciones del Papa eran una oportunidad de discutir ampliamente sobre el tema. El exministro expresó que callar la voz del Papa «es bien absurdo y opuesto a nuestras más elementales tradiciones culturales y democráticas», para luego confrontar a las guías, señalando que su contenido era un ataque directo a la institución conyugal, una puerta abierta a la permisividad y promiscuidad sexual y una afrenta a la consciencia moral y cristiana del pueblo. ${ }^{37}$ El mismo Brenes Jiménez, en una intervención posterior, se preguntaba quién era el que realmente se entrometía en los asuntos de Costa Rica, y concluía que en lugar del Papa, eran los organismos internacionales que habían promovido el proyecto. Organismos que — según el exministro- estaban basados en una concepción de la sexualidad «radicalmente primitiva y obsoleta» con un «carácter genitalista y animalesco». ${ }^{38}$ Román Arrieta y Helena Ospina compartían la posición de Brenes. ${ }^{39}$ Un ciudadano afirmó que estaban «redactadas e ilustradas en forma tal que inducen a la sexualidad desenfrenada entre hombres lascivos y mujeres con furor uterino». 40

En el otro lado de la moneda de estas afirmaciones, personajes como Virginia Beckles, presidenta de la Asociación Demográfica Costarricense (ADC); William Vargas, director de medicina preventiva de la Caja Costarricense de Seguro Social (CCSS); Rolando Zamora, director del

\footnotetext{
${ }^{36}$ Emilia Mora Gamboa, «Interpretación errónea a mensaje de Juan Pablo II», La Prensa Libre (San José), 22 noviembre de 1991, 1.

37 Víctor Brenes Jiménez, «A la palestra sin miedo», La Nación (San José), 22 noviembre de 1991, 18.

38 Víctor Brenes Jiménez, «¿Se entromete el Vaticano en Costa Rica?», La República (San José), 26 noviembre de 1991, 14.

${ }^{39}$ Ronald Espinoza, «Pugna por orientación de la educación sexual», La Nación (San José), 25 noviembre de 1991, 5.

${ }^{40}$ Mario Borges, «Oportuna intervención del Papa», La Prensa Libre (San José), 27 noviembre de 1991, 2.
} 
Centro Nacional de Didáctica; Emilia María León, directora general de Salud; Efraín Valerio, coautor, entre muchos otros profesionales y figuras públicas, salieron en defensa de los esfuerzos estatales por institucionalizar la educación sexual y la elaboración de los documentos. ${ }^{41}$

\section{IMPUGNACIÓN RELIGIOSA}

Originalmente la CECOR había propuesta tres sugerencias para complementar el contenido de las guías, no obstante, después de las declaraciones del Papa, estas sugerencias originales parecía que ya no eran suficientes, se empezó a considerar que la introducción de un marco teórico doctrinal como un anexo no resolvía las deficiencias de fondo. En ese sentido, Román Arrieta afirmó en noviembre de 1991 que iba a proponer la reelaboración completamente nueva. Aunque esta propuesta fue, en un inicio, a título personal de Arrieta, prontamente sería acuerpada por la CECOR. ${ }^{42}$ Debido a lo anterior, para Efraín Valerio, coautor, las declaraciones de Arrieta fueron desconcertantes y de alguna manera se contraponían con el compromiso adquirido por los obispos con el MEP.

En respuesta a Valerio, Arrieta aceptó que en un primer momento solamente se dieron tres sugerencias, para luego manifestar que, bajo las nuevas circunstancias, la Iglesia se oponía. ${ }^{43}$ Esto produjo que estas entraran, nuevamente, en un periodo de espera, mientras la CECOR acordaba cuál sería su veredicto. Para Marvin Herrera, ministro de Educación, si todo se desarrollaba de acuerdo con lo planeado, se podría iniciar con su en el primer semestre de 1992, sin embargo, no sería hasta diciembre de 1991 que los obispos se reunirían para discutir el destino de este proyecto. ${ }^{44}$

La CECOR arribó a la conclusión de que era necesario confeccionarlas desde cero, al considerar que en las actuales se presentaban «abundancia de mensajes subliminales que favorecen el uso indiscriminado del

\footnotetext{
${ }^{41}$ Ronald Espinoza y Gabriela Quirós, «Urgen políticas de educación sexual», La Nación (San José), 24 noviembre de 1991, 8.

42 María Isabel Solís, Ronald Espinoza y Carlos Arguedas, «Arzobispo propone nuevas guías», $L a$ Nación (San José), 23 noviembre de 1991, 4.

${ }_{43}$ Emilia Mora Gamboa, «Efraín Valerio no tiene toda la razón», La Prensa Libre (San José), 27 noviembre de 1991, 3 .

${ }_{44}$ Mariana Lev, «Se enseñará sexualidad con una base moral y espiritual», La República (San José), 24 noviembre de 1991, 4.
} 
sexo», ${ }^{45}$ y la ausencia de referencias al sacramento del matrimonio. Los obispos confirmaron su disposición para elaborar los nuevos documentos y aseguraron que contaban con la colaboración de médicos, juristas, pedagogos y demás profesionales con una profunda visión religiosa. ${ }^{46}$ A raíz de la decisión de la CECOR, el presidente Rafael Ángel Calderón ordenó su elaboración, confirmando que "hemos sido muy claros en cuanto a nuestro deseo de que las nuevas guías sexuales surjan del consenso entre el gobierno y la Iglesia Católica».47

El arzobispo, Román Arrieta, publicó una carta en diferentes medios de comunicación, dirigida al ministro de Educación Pública, en donde se enumeraban las razones que llevaron a la CECOR a sugerir la reelaboración. En la carta se mencionaba que, por falta de una adecuada educación, eran muchos los jóvenes que se pervierten, se afirmaba la necesidad de que los padres de familia ocuparan el legítimo lugar para impartir esta educación y que, debido a su incapacidad, dicha tarea debía recaer subsidiariamente en la escuela. Se coincidía con que la falta de una educación había provocado relaciones sexuales irresponsables, asimismo, se concordaba en que la educación debía basarse en valores morales de vigencia universal, válidos no solo para católicos, sino para todo tipo de creyentes y no creyentes. ${ }^{48}$

La CECOR llegó a la conclusión de que, más que un documento aparte, era necesario integrar estas observaciones en el tejido mismo de los documentos, evitando que los profesores hicieran caso omiso del marco teórico doctrinal por tratarse de un anexo, y garantizar que, en el abordaje de los temas, la postura de la Iglesia fuera simultánea. Las metodologías pedagógicas originales fueron valoradas positivamente y no se propusieron cambios en ese sentido.

En este proceso de redacción y discusión, empezaron a surgir desacuerdos en torno al proyecto político más adecuado para construir y

\footnotetext{
45 José Miguel Fonseca, «Iglesia veta guías sexuales», La Nación (San José), 18 diciembre de 1991, 5.

46 José Miguel Fonseca, «Iglesia veta guías sexuales», 5.

47 María Solís y Mauricio Martínez, «Calderón ordena nuevas guías sexuales», La Nación (San José), 19 diciembre de 1991, 5.

${ }^{48}$ Román Arrieta, «Por nuestra juventud», La Nación (San José), 19 diciembre de 1991, 18; Román Arrieta, «Por nuestra juventud», Eco Católico (San José), 5 enero de 1992, 4.
} 
regular la normalidad sexual. Por un lado, el Estado y las autoridades educativas y, por otro, la Iglesia y sus seguidores. En medio de esta pugna de saberes y posiciones, los medios de comunicación funcionaron como una plataforma para que distintas personas e instituciones mostraran su posición, a favor o en contra, de estos proyectos políticos. A inicios de la década de 1990 la Iglesia mantiene una posición hegemónica frente a los otros proyectos, y logra imponer su visión acerca de cómo se debe construir y regular la normalidad sexual en las aulas. No obstante, dicha hegemonía por parte de la Iglesia fue resistida y refutada por varios actores sociales, como se verá a continuación.

\section{LOS DISCURSOS EN CONTRA Y A FAVOR DE LA INTERVENCIÓN DE LA CECOR EN LAS GUÍAS DIDÁCTICAS}

Efraín Valerio admitió que, aunque no se incluían valores cristianos, sí contaban con valores humanistas universales, y rechazó que hubiese mensajes subliminales. Para el experto en biología y sexualidad, este viraje repentino de los obispos era una señal clara de ciertas influencias provenientes de los sectores más extremistas de la Iglesia y el Vaticano, criticaba la facilidad con la que habían cambiado de opinión en relación con estas. Más de 10.000 unidades habían sido impresas a un costo millonario, y la ligereza con que estas estaban siendo desestimadas era evidencia de una "apetencia de imponer criterios a rajatabla. Aunque somos cristianos, nuestras guías por la naturaleza de nuestro trabajo no son un catecismo cristiano, ni pueden serlo».49

Con relación a la omisión del concepto de matrimonio Valerio afirmó que la Comisión Redactora, cuyos integrantes incluían a varios sacerdotes, consideró adecuado el término de pareja ya que era más realista, y porque el matrimonio por sí solo no garantizaba buenas condiciones de relación conyugal. En miras de las acusaciones sobre mensajes subliminales, las calificó de calumnias y difamación. ${ }^{50}$ Para él, el país estaba en presencia de una lucha ideológica, «nuestros opositores desean que las guías prediquen recetas morales a los jóvenes y que los profesores los

\footnotetext{
49 Efraín Valerio Charpentier, "Paranoia cristianoide», La Prensa Libre (San José), 23 diciembre de 1991, 9.

50 Efraín Valerio Charpentier, «Paranoia cristianoide», 9.
} 
adoctrinen. Nosotros procuramos la autonomía y la autodeterminación auténticas de los jóvenes». Una de las principales críticas de Valerio frente las críticas de la CECOR, era que el trabajo y la participación de los sacerdotes que estuvieron involucrados en la redacción original no parecía ser considerado por los obispos, y el hecho de proponer un nuevo proyecto era interpretado como «un despropósito, una exageración inadmisible, un auténtico espécimen de intromisión en una tarea que no es de su competencia». ${ }^{51}$ Para el ministro de Educación, el hecho de que la CECOR rechazara los documentos en cuya elaboración habían participado sacerdotes designados por esa misma Conferencia, era evidencia de que la Iglesia estaba cediendo ante grupos ortodoxos de su estructura. ${ }^{52}$

Para el exdiputado Javier Solís, la materia sexual no era precisamente uno de los temas de mayor competencia en los círculos clericales, lo cual influía en la visión represiva que de esta institución emanaba. ${ }^{53}$ En un artículo posterior, Solís manifestó que la sexualidad era una materia difícilmente comprensible a través de la simple exposición teórica, siendo la experiencia directa algo imprescindible. ${ }^{54}$ De igual manera, en otro texto aducía que no eran precisamente los católicos más comprometidos con las causas sociales los que se habían levantado en contra, al mismo tiempo que reprochaba la actitud de los gobernantes «perezosos, mojigatos o irresponsables, que le tienen miedo a las sotanas».55 Para el autor, el veto interpuesto por la Iglesia no tenía razón de ser, ya que violaba los principios constitucionales del país.

Otros argumentos apuntaban a que la Iglesia se había convertido en el cuarto poder del Estado, cuyo accionar «ha finalizado con el espectáculo lamentable de los representantes del Poder Ejecutivo doblando la rodilla y bajando la cabeza». ${ }^{56}$ En un artículo que ocupó la portada de La Nación, la ministra interina de educación, Virginia Rojas Arroyo, manifestó que en el

\footnotetext{
${ }^{51}$ Efraín Valerio Charpentier, «Verdolaga hipertrofiada», La Prensa Libre (San José), 28 diciembre de 1991, 2.

52 Laura Valverde y Ángela Orozco, «Iglesia cedió ante grupos ortodoxos», La República (San José), 7 enero de 1992, 2.

53 Javier Solís, «Los sacerdotes saben poco de sexo», La República (San José), 10 enero de 1992, 9.

${ }^{54}$ Javier Solís, «Sexo, Iglesia y represión», La República (San José), 22 enero de 1992, 2.

55 Javier Solís, «Un derecho que no existe», La República (San José), 31 enero de 1992, 25.

${ }^{56}$ Hugo Mora Poltronieri, «Estado y religión oficial», Universidad (San José), 17 enero de 1992, 4.
} 
curso lectivo que iniciaba en 1992, iba a ser imposible aplicar las guías didácticas, ya que, si en las anteriores se había trabajado por cinco años y fueron rechazadas, con las nuevas era incierto cuánto se iba a durar en completarlas. El MEP contaba con pocos para empezar este nuevo trabajo, y aunque las originales seguían en las bodegas del Ministerio, siendo estudiadas por el Consejo Superior de Educación, su uso no sería efectivo a menos que el presidente ordenara ponerlas en circulación. ${ }^{57}$

El secretario general del Sindicato de Educadores Costarricenses (SEC), Edilberto Escobar Cascante, afirmó que iba a exigirle al MEP que las entregara oficialmente a los maestros para que pudieran empezar a ser utilizadas en el ciclo lectivo, a raíz de la urgencia con la que vislumbraba su uso en la educación. ${ }^{58}$ Por su parte, el sexólogo Javier Ortiz Gutiérrez manifestó que la información contenida en estas estaba actualizada, era adecuada para los diferentes grupos de edad meta, eran exhaustivas y no inducían a la imposición como estrategia pedagógica, asimismo, al igual que Valerio, Ortiz descartaba la presencia de mensajes subliminales, afirmando que "todo aquel que vea en ellas un "mensaje subliminal" erótico subversivo, lo que está viendo en realidad es el reflejo de su propia patología sexual».59

Las críticas a la posición de la Iglesia se intensificaban conforme llegaba el momento de inicio de las clases. Por ejemplo, se expuso que «dejarle la educación sexual a la Iglesia, es como pedirle a un vegetariano su opinión sobre la carne roja, o a un abstemio su opinión sobre el licor. El sentido común [...] indica que para hablar de algo hay que conocerlo». ${ }^{60}$ Se manifestó que el oscurantismo propio de la Edad Media había vuelto a Costa Rica por medio de la acción desarrollada por la Iglesia. Había un lamento por todos los recursos desperdiciados y por la actitud del gobierno, que desechaba criterios profesionales por pensamientos moralistas. Se alegó que «el único perjudicado es sin duda el

\footnotetext{
${ }^{57}$ Ronald Espinoza, «Postergan educación sexual», La Nación (San José), 21 enero de 1992, 5.

${ }_{58}$ Emilia Mora Gamboa, «SEC exigirá uso de guías sexuales», La Prensa Libre (San José), 22 enero de1992, 6 .

59 Javier Ortiz Gutiérrez, «En defensa de las "guías sexuales"», Universidad (San José), 24 enero de 1992, 6.

${ }_{60}$ Dino Starcevic, «iAsí no se vale!», La República (San José), 29 enero de 1992, 22.
} 
joven quien pese a ser el único encargado de manejar sus necesidades sexuales, ahora se ve privado de la orientación sexual adecuada».61

El presidente de la Federación de Estudiantes de la Universidad de Costa Rica (FEUCR), Alberto Cortés, increpaba al Estado por ceder ante la campaña de corte moralista dirigida por los sectores más retrógrados de nuestra sociedad, se tildaba al gobierno de irresponsable y negligente por obstaculizar la aplicación de las guías didácticas. ${ }^{62}$ Asimismo, hubo gremios que también alzaron la voz, entre ellos el Colegio de Psicólogos de Costa Rica consideraba que estas eran adecuadas, bien desarrolladas y estimulantes para la enseñanza de la sexualidad con seriedad y humanismo. ${ }^{63}$

Mediante un análisis histórico sobre la relación entre el Estado y la religión, el académico Claudio Vargas argumentó que «la bendición eclesiástica sigue siendo un preciado medio que gobernantes y líderes en general, buscan, con el propósito de identificarse con los mismos valores que los de la mayor parte de la sociedad».64 Por su parte, el teólogo Gerardo Esquivel Monge, las colocó en la balanza, afirmando que eran obras didácticamente bien concebidas, alejadas de dogmatismos, y reflexivas, lo cual lo llevó a expresar que «en este caso la jerarquía eclesiástica se excedió en su intransigencia». ${ }^{65}$ En una apreciación similar, para el psicólogo y teólogo de la ADC, Simón Benjamín, la Iglesia «parcializa la realidad, porque los jóvenes van a buscar ese vacío en la calle y muchas veces obtienen consejos equivocados».66

En respuesta a todas estas voces que criticaban el accionar de la Iglesia, Román Arrieta reafirmaba que los documentos tenían problemas de fondo que desorientaban la vida sexual de los adolescentes, al mismo tiempo que negaba la posibilidad de que la Iglesia brindara ayuda económica

\footnotetext{
${ }^{61}$ Basilio Quesada Chanto, «Sexo, mentiras y juventud», La República (San José), 30 enero de 1992, 22.

62 Alberto Cortés, «Las guías sexuales y la realidad costarricense», La República (San José), 7 febrero de 1992, 22; Alberto Cortés, «FEUCR respalda guías sexuales», Universidad (San José), 7 febrero de 1992, 18.

${ }_{63}$ Adriana Pereira, «Psicólogos a favor de guías sexuales», Universidad (San José), 7 febrero de 1992, 18.

${ }^{64}$ Claudio Vargas, «La Iglesia, el Estado y las guías sexuales», Universidad (San José), 13 marzo de 1992,6 .

${ }^{65}$ Gerardo Esquivel Monge, «Sexualidad y educación», Universidad (San José), 5 junio de 1992, 5.

${ }^{66}$ Milena Fernández, "Guías sexuales de la mano con la polémica», La Nación (San José), 7 marzo de 1993, 6.
} 
en la reedición de estos, aseguraba que «por principio no podríamos dar ni un cinco porque no hemos cometido ninguna falta, porque no se consultó antes de editarlas», 67 aunque como ya se vio anteriormente, en la elaboración inicialmente hubo sacerdotes seleccionados por la misma CECOR.

En un artículo explícitamente dirigido a Efraín Valerio, se declaraba que la Iglesia no rehuía de la educación sexual, pero exigía una formación humana que, según el autor, no estaba presente. Terminaba su intervención exponiendo que "aquí no hay ninguna inquisición: estamos en una sociedad pluralista, y el que quiera tiene pleno derecho de sostener posturas no cristianas, sin temor a la hoguera [...] es el Prof. Valerio quien encabeza la procesión [...] de toda una ideología fraguada por organismos foráneos». ${ }^{68}$ La opinión pública, desplegada por los medios de comunicación, hizo evidente que en el país habían sectores opuestos en relación a la injerencia que debía ejercer la Iglesia dentro de las políticas educativas vinculadas con la educación sexual. Tanto de un lado como del otro, el argumento de que poderes externos estaban manejando los hilos de la política era recurrente.

\section{LAS GUÍAS DIDÁCTICAS REFORMULADAS}

La reelaboración de las guías a partir de las observaciones de la CECOR fue un proceso más rápido, ya que se contaba con las bases de las originales. Para Arrieta, el proceso no iba a tomar más de cuatro meses. ${ }^{69}$ Efectivamente, para mayo de 1992, la comisión especial designada por la CECOR terminó el estudio con los seis obispos del país; dicho trabajo consistió en una revisión total de los documentos y la comisión estuvo integrada por los sacerdotes Víctor Hugo Munguía, Rodolfo Murillo y José Alberto Quirós, junto con los laicos Julián Zamora Dobles, María Eugenia Dengo , Miguel Ángel Campos y Álvaro Rivera. ${ }^{70}$ Llegar a ese punto no fue fácil, ya que además de la intensa actividad mediática

\footnotetext{
${ }^{67}$ Emilia Mora, «Arzobispo denuncia pérdida de valores», La Nación (San José), 6 enero de $1992,6$.

68 Carlos Alonso Vargas, «Mitología valeriana», La República (San José), 6 febrero de 1992, 22.

69 Emilia Mora, «Reelaboración de guías sexuales será rápida», La Prensa Libre (San José), 21 enero de 1992, 4.

70 Ronald Espinoza, «Ratifican uso de guías sexuales», La Nación (San José), 3 mayo de 1992, 4.
} 
a favor y en contra la intervención de la Iglesia, el mismo Consejo Superior de Educación (CSE) sostuvo que era de su competencia analizarlas, ya que en vista de la ausencia de un programa nacional de educación sexual, los folletos venían a cumplir ese papel y, por lo tanto, el CSE debía darles su aprobación. ${ }^{71}$

La CECOR afirmó que entregaría los documentos revisados con el tiempo suficiente para que fueran puestos en funcionamiento durante el segundo ciclo lectivo del año, no obstante, esto no sucedió y fue hasta el primer ciclo de 1993 que finalmente se logró la entrega a los y las docentes. En relación con las modificaciones, si llegar a un consenso permitía una puesta en funcionamiento más expedita, el MEP estaba dispuesto a aceptarlas. ${ }^{72}$ Después de siete años de elaboración y discusión, las guías estaban a punto de salir a la luz.

Uno de los cambios más discutidos fue el de las ilustraciones eliminadas. Para la CECOR, debían tener una misión más formativa que instructiva, por lo que consideraron que varias de las imágenes presentes a lo largo de las tres eran innecesarias o reiterativas. ${ }^{73}$ En relación con la inclusión del condón como un método de planificación familiar, la Iglesia sostenía su oposición, aunque mantuvo dichos elementos. Arrieta expresó, en ese sentido, que «el condón fomenta las relaciones sexuales prematrimoniales, su uso es irresponsable porque no resulta ciento por ciento seguro, más bien ha servido para propagar el SIDA».74 Para el discurso religioso, la regulación de la normalidad sexual pasaba por filtros mucho más específicos que tenían que ver con su negativa a aceptar dentro de su doctrina, métodos anticonceptivos artificiales.

En un editorial publicado por el presbítero Armando Alfaro Paniagua en el Eco Católico, se afirmaba contundentemente que: «el problema más álgido de la sociedad humana en finales de este siglo se llama

\footnotetext{
${ }^{71}$ Emilia Mora Gamboa, «Pugna por aprobación de las guías sexuales», La Nación (San José), 26 febrero de 1992, 4.

${ }^{72}$ María Isabel Solís, «Iglesia aplaza entrega de guías sexuales», La Nación (San José), 8 junio de 1992, 4.

${ }^{73}$ Laura Martínez, «Suprimen dibujos de guías sexuales», La República (San José), 12 julio de 1992, 5.

${ }^{74}$ Milena Fernández, «Iglesia sugiere fuertes cambios en guías sexuales», La Nación (San José), 6 agosto de 1992, 4.
} 
"comportamiento sexual" ». ${ }^{75}$ De acuerdo con esta tesis, aspectos como la crisis económica, la corrupción política, la contaminación ambiental o los conflictos militares ocupaban un lugar secundario frente a la problemática de la sexualidad. La afirmación del presbítero evidencia cómo, dentro de este contexto, la sexualidad tenía un papel de banda transbordadora, sobre la cual se movían toda una serie de ansiedades sociales que no encontraban una válvula de escape adecuada. ${ }^{76}$ Dentro del editorial se enmarcó el contexto de forma polarizada, por un lado, los provocadores de la libertad sexual y, por otro lado, la mayoría, que todavía están con Dios, y la pugna entre ambos sectores, entre ambos proyectos políticos sobre la normalidad sexual, recaía en esta ocasión en el tema de las guías didácticas.

Para inicios de 1993 se confirmó estaban listas para ser distribuidas entre los docentes de séptimo, octavo y noveno del sistema educativo público. En retrospectiva, el ministro de Educación manifestó que, después de los cambios, se habían respetado en un $90 \%$ los contenidos originales, tratando de balancear la postura de la Iglesia con los principios científicos. En el siguiente cuadro se sistematizan los cambios.

\section{Cuadro 1. Cambios elaborados por la CECOR en las guías didácticas de educación sexual}

\begin{tabular}{|c|l|}
\hline Guía & \multicolumn{1}{c|}{ Principales cambios } \\
\hline Séptimo & $\begin{array}{l}\text { 1: Se amplía el concepto de adolescencia, ya que, además de ser (en la } \\
\text { original) una etapa de cambios se agrega que, también, es una época para } \\
\text { adherirse a escalas de valores y principios cívico y morales cristianos } \\
\text { 2: Cuando se habla de la definición científica del semen, en las nuevas se } \\
\text { agrega que la madurez biológica siempre tiene que ir acompañada con la } \\
\text { madurez psicológica y social }\end{array}$ \\
$\begin{array}{l}\text { 3: Se censuran las relaciones sexuales prematrimoniales entre } \\
\text { adolescentes, y se enfatiza en que la sexualidad debe ser ejercida de } \\
\text { manera responsable, satisfactoria y consciente }\end{array}$ \\
$\begin{array}{l}\text { 4: Se introduce la idea de que el hombre y la mujer fueron creados a } \\
\text { imagen y semejanza de Dios. } \\
\text { 5: Tanto la pornografía como la violencia son conceptualizadas como } \\
\text { agresiones morales }\end{array}$ \\
\hline
\end{tabular}

\footnotetext{
75 Armando Alfaro Paniagua, «Guías de sexualidad humana», Eco Católico (San José), 9 agosto de 1992, 8.

76 Jeffrey Weeks, Sexuality (London: Routledge, 2003), 102.
} 


\begin{tabular}{|c|c|}
\hline Guía & Principales cambios \\
\hline Octavo & $\begin{array}{l}\text { 1: En las originales se explica de forma detallada, a lo largo de } 20 \\
\text { páginas, el significado, ventajas y desventajas de múltiples métodos } \\
\text { anticonceptivos artificiales, en las nuevas, en } 6 \text { páginas se explica de } \\
\text { forma bastante resumida y sin entrar en detalles, la función y el } \\
\text { significado de dichos métodos } \\
\text { 2: Se impugna la noción de relaciones sexuales en el marco de «formas } \\
\text { novedosas de placer» y, en su lugar, se profundiza en que las relaciones } \\
\text { deben enmarcarse en el amor y el respeto } \\
\text { 3: En las originales se afirmaba que las relaciones sexuales entre parejas } \\
\text { no necesariamente se daban para engendrar hijos, en las nuevas se } \\
\text { cambia esto y se establece que la procreación es el objetivo de las } \\
\text { relaciones } \\
\text { 4: Se insiste en que la procreación de un hijo no es el resultado de una } \\
\text { entrega irracional, sino de una acción responsable y consciente } \\
\text { 5: En las originales se brindaba información acerca de } 15 \text { razones } \\
\text { sociales por las cuales una mujer podía recurrir al aborto, esto se } \\
\text { sustituyó por una visión moral de la Iglesia sobre el tema, enfatizando las } \\
\text { sanciones legales }\end{array}$ \\
\hline Noveno & $\begin{array}{l}\text { 1: Se omite la afirmación de que la masturbación es algo normal } \\
\text { 2: Se colocó al inicio de cada asunto a tratar, una cita bíblica relacionada } \\
\text { 3: En la imagen que ilustraba situaciones de la vida real donde aparecía } \\
\text { una joven siendo regañada por sus padres, se cambió por la imagen de } \\
\text { dos jóvenes con sus padres sonrientes } \\
\text { 4: Cuando se habla de paternidad responsable, se sustituyó el término } \\
\text { "pareja» por «esposos» }\end{array}$ \\
\hline
\end{tabular}

Fuente: Laura Martínez, «Guías sexuales saldrán sin mayores cambios», La República (San José) 27 enero de 1993, 2; Marilú Papili, «MEP aceptó las recomendaciones», La Prensa Libre (San José), 28 agosto de 1992, 17; Fernández, «Iglesia sugiere fuertes cambios en guías sexuales», 4; Martínez, «Suprimen dibujos de guías sexuales», 5; Milena Fernández y Ronald Matute, «Leves cambios en guías sexuales», La Nación (San José), 3 agosto de 1992, 8; Fernández, "Guías sexuales de la mano con la polémica», 6; Milena Fernández, «Énfasis moral predomina en los textos educativos», $L a$ Nación (San José), 25 febrero de 1993, 8. Queda pendiente un análisis empírico del contenido total de las guías didácticas.

Como se puede observar en el cuadro anterior, los cambios fueron bastante específicos, y se enfatizaron en elementos relacionados principalmente con la anticoncepción. Elementos como la masturbación y la pornografía adquirieron un tinte negativo e incluso patológico con las nuevas, además de que hubo un claro enfoque en el tema del matrimonio y la 
procreación. En las originales, se detallaban las ventajas y desventajas de siete métodos de planificación artificiales y tres naturales, se destacaba el porcentaje de confiabilidad de cada uno, asimismo se hablaba ampliamente del aborto y en las ilustraciones se mostraban penes erectos y adolescentes embarazadas, además de primeros planos sobre partes del cuerpo humano.

En las nuevas todo eso se modificó, los métodos de planificación fueron abordados de forma escueta, se enfatizó que el único método aprobado por la Iglesia era la abstinencia, con el aborto se eliminaron las posibles razones, pero se reforzó el tema de las sanciones legales y, finalmente, varias ilustraciones fueron eliminadas o cambiadas. Las guías entraron en funcionamiento en 1993, y como paso previo, se capacitaron a más de 1900 profesores de religión, ciencias y educación familiar para afinar la utilización de los textos. Desde ese primer momento, una de las principales limitantes se hizo evidente; las dudas acerca de los prejuicios sexuales de los y las docentes.

\section{LA APLICACIÓN DE LAS GUÍAS DIDÁCTICAS}

Las décadas de 1980 y 1990 fueron de cambios importantes para el sistema educativo costarricense. ${ }^{77}$ Por ejemplo, en 1995 hubo una manifestación por parte de los educadores en oposición a la reforma de ley de pensiones, dicha manifestación fue reprimida de manera bastante fuerte por las fuerzas policiales y el gobierno, provocando una mayor intensificación del descontento con la clase política. En general la recuperación de la inversión educativa se desarrolló de manera bastante lenta, la inversión estatal en educación para la década de 1990 estaba, en términos proporcionales, por debajo de los niveles de 1979,

\footnotetext{
77 En un contexto de crisis económica durante la década de 1980, impulsada por múltiples factores externos e internos, los organismos financieros internacionales como el Fondo Monetario Internacional y el Banco Mundial establecieron una serie de condiciones para acceder a créditos y préstamos. El ajuste estructural que se llevó a cabo en Costa Rica implicaba la reducción y reorientación del gasto estatal, la liberalización económica y la privatización de empresas públicas. Esta reorientación neoliberal del modelo de desarrollo tuvo efectos en la educación pública, específicamente en el deterioro de los salarios de docentes y la deserción estudiantil. Para un análisis detallado de los efectos de la crisis y el ajuste estructural en las políticas educativas de Costa Rica, ver: Martin Carnoy y Carlos Torres, Educational change and structural adjustment: a case study of Costa Rica. (París: UNESCO, 1992), 1-56. Para un análisis del papel del Banco Mundial en las políticas educativas latinoamericanas: Xavier Bonal, «Globalización y política educativa: un análisis crítico de la agenda del Banco Mundial para América Latina», Revista Mexicana de Sociología, vol. 64, n. 3 (2002).
} 
y sería hasta el 2000 que se logró finalmente alcanzar esa marca nuevamente. ${ }^{78}$

Los salarios de docentes también tendieron a la baja durante los años noventa, con métodos de enseñanza que, incluso llegando a inicios del siglo XXI, seguía basándose en la memorización y la aplicación mecánica de procedimientos, con muy pocas oportunidades para motivar un pensamiento más innovador y abstracto. Profesionalmente, hubo un cambio importante en la dinámica de la formación de docentes, el porcentaje de los títulos de educación expedidos por universidades privadas aumentó de 10\% a 47\% entre 1990 y 1999, eso significaba que el Estado tenía poco control sobre la calidad y la preparación del cuerpo docente, dando paso a una formación más pedagógica y menos académica. ${ }^{79}$

Estos elementos del contexto educativo nacional permiten entender, de forma más clara, el destino de las guías didácticas y el papel que tuvieron propiamente en las aulas y en las estrategias de las personas docentes a lo largo de la segunda mitad de la década de 1990, destino fraguado en un escenario donde había una deficiente y poco fiscalizada preparación académica, un alto nivel de resistencia al cambio, un vínculo fuerte entre educación e Iglesia, y la falta de recursos por parte del MEP para dar capacitaciones sistemáticas y efectivas.

Iván Molina argumenta que, una vez que el anticomunismo perdió fuerza a finales de la década de 1980, los grupos conservadores, bajo el liderazgo de las iglesias cristianas, ${ }^{80}$ empezaron a concentrar sus

\footnotetext{
${ }^{78}$ Molina, La educación en Costa Rica, 487-495.

${ }_{79}$ Molina, La educación en Costa Rica, 518-524.

${ }^{80}$ En 1986 entra en la arena electoral la Alianza Nacional Cristiana, primer partido evangélico costarricense. En 1998, el Partido Renovación Costarricense logra una diputación por la provincia de San José. Para las elecciones presidenciales del 2018, el Partido Restauración Nacional casi llega al Poder Ejecutivo, además logra cuadriplicar su número de congresistas. Entre los rasgos más comunes que se observan en este tipo de diputados está el voto corporativista, el énfasis de los valores cristianos dentro de la política, la defensa del «orden natural» fundamentado en la familia nuclear heterosexual, así como la movilización de masas por medio de representaciones populistas de supuesta transparencia moral, ver: Laura Fuentes Belgrave, «Politización evangélica en Costa Rica en torno a la agenda «provida»: ¿Obra y gracia del Espíritu Santo? », Revista Rupturas, vol. 9, n. 1 (2019): 86-89. https://doi.org/10.22458/rr.v9i1.2230. La creciente polarización sobre el tema de la educación sexual en los últimos años en Costa Rica está enmarcada en la creciente influencia del neopentecostalismo en la arena pública, así como un recrudecimiento del conservadurismo frente a legislaciones recientes vinculadas con la fecundación in vitro, el matrimonio igualitario y la norma técnica sobre el aborto terapéutico.
} 
esfuerzos en atacar cualquier síntoma de secularización social, entre ellos la libertad sexual, generando un contexto favorable para la influencia de la jerarquía católica en la enseñanza, así como la apertura de instituciones educativas religiosas, una expansión creciente de la industria espiritual y formación de partido políticos liderados por pastores evangélicos. ${ }^{81}$

Es sobre ese trasfondo que se empezaron a aplicar, aunque más bien habría que decir que en la mayoría de las ocasiones, las guías fueron ignoradas o quedaron en el olvido. La sospecha de Valerio en 1993 se hizo patente en los años siguientes; explicar temas sexuales a adolescentes era una tarea que los adultos no sabían cómo abordar. En un foro de discusión, el sexólogo Javier Ortiz, Efraín Valerio y Simón Benjamín de la ADC afirmaron que los adultos debían superar un sinnúmero de prejuicios, ascos, y actitudes vinculadas con la cultura de sexofobia presente en el país. ${ }^{82}$ Posición defendida varios años después por los mismos sujetos, agregando que la desinformación provocaba mitos y tabúes que incidían en el aumento de violaciones, embarazos no deseados y relaciones sexuales genitalizadas. ${ }^{83}$ En una encuesta solicitada por La Nación, se evidenció que los documentos eran prácticamente desconocidos por los estudiantes, ${ }^{84}$ a pesar de que ya llevaban más de 8 meses de haber entrado en circulación.

En el sector educativo privado la situación variaba mucho, colegios como el Lincoln tenían un plan de formación humana de séptimo hasta duodécimo que, según sus desarrolladores, había sido exitoso. Una declaración de un estudiante de 14 años fue bastante reveladora sobre el nivel de contradicciones que se podían dar al interior de los colegios, «el orientador me dice que me masturbe todo lo que quiera; la profesora de ciencias, que lo haga, pero no mucho porque puedo quedar impotente; $y$ el de religión que es el pecado más grande». ${ }^{85}$ Incluso habían planes para instaurar un programa de educación sexual en edad preescolar, con un

\footnotetext{
81 Molina, La educación en Costa Rica de la época colonial al presente, 53.

${ }^{2}$ Ivannia Varela Quirós, "Adultos deben enfrentar su analfabetismo sexual», La República (San José), 30 mayo de 1993, 2.

${ }^{83}$ María Isabel Solís, «Papi, hablemos de sexo», La Nación (San José), 28 abril de 1997, 1B-2B.

${ }^{84}$ Gina Polini, «Claras confesiones», La Nación (San José), 1 octubre de 1993, 1B.

85 Gina Polini, «Sexo desde la pizarra», La Nación (San José), 3 octubre de 1993, 1B.
} 
plan piloto implementado en el Centro Infantil de San Antonio de Guadalupe. ${ }^{86}$ A raíz de estos nuevos esfuerzos, la Iglesia mantenía su posición de fiscalización sobre cualquier iniciativa estatal dirigida a institucionalizar la educación sexual en otros niveles. Por ejemplo, cuando se empezó a gestar la redacción de las guías para la educación primaria, nuevamente se argumentó sobre la necesidad de una «inspiración religiosa». ${ }^{87}$

\section{CONCLUSIÓN}

Poco a poco, mediante un proceso paulatino que asemejaba una candela al viento, el impacto y el efecto de las guías sexuales fue disminuyendo, y conforme pasaban los meses, las acaloradas discusiones, las polémicas y las posiciones encontradas fueron atenuándose, teniendo una presencia más limitada en la prensa. De alguna forma, ese proceso iba aparejado con una invisibilización del tema en las mismas aulas. Un artículo de La República manifestaba que, después de cuatro años, su aplicación continuaba a la deriva, a causa de los prejuicios de los docentes y su falta de capacitación. Valerio confirmó sus sospechas nuevamente, eran pocos los docentes que las utilizaban activamente en las clases, y esto se debía también a «desconocimiento, los escrúpulos, sistemas morales trasnochados e inseguridad». ${ }^{88}$

A pesar de que el tema de la sexualidad era abordado dentro de varias materias como ciencias, educación para el hogar y religión, el uso de las guías era optativo, es decir, su aplicación no se había hecho obligatoria, eran materiales de referencia. ${ }^{89}$ Por lo tanto, no había medios para fiscalizar si estaban siendo utilizadas. Cuando los profesores las recibían, también se les entregaba un documento en el cual se destacaba la importancia de instruir a los alumnos sobre el tema. ${ }^{90}$

Las estadísticas eran lapidarias, para 1997 más de un 50\% de las personas jóvenes no había recibido información sobre educación sexual en

\footnotetext{
${ }^{86}$ Gina Polini, «Sexualidad en la lonchera», La Nación (San José), 18 diciembre de 1993, 1 B.

${ }^{87}$ Milena Fernández, «Iglesia pide completa revisión de guías», La Nación (San José), 23 marzo de 1994, 19.

${ }^{88}$ Rocío Pastor, «Guías sexuales a la deriva», La República (San José), 1 enero de 1996, 4-5.

${ }^{89}$ Ronald Espinoza, «Sexualidad no obligatoria», La Nación (San José), 26 julio de 1996, 1B.

90 Pastor, «Guías sexuales a la deriva», 4-5.
} 
las aulas. ${ }^{91}$ Los mismos obstáculos que se habían detectado al inicio de su aplicación seguían vigentes años después, los mitos y prejuicios de los docentes tenían un papel protagónico. ${ }^{92}$ En un editorial de La República en 1999, se afirmaba que, aunque la pugna entre el MEP y la Iglesia se había estabilizado, el nuevo problema era la poca preparación de los docentes para aclarar dudas, se planteaba la sugerencia de que el MEP impartiera talleres de capacitación. ${ }^{93}$ Es revelador que para 1997, directores y docentes de diferentes institutos como la Escuela León XIII, la Escuela José Figueres Ferrer y el Colegio de Coronado afirmaran que nunca habían recibido las guías, lo cual era confirmado por la Asociación Nacional de Educadores (ANDE), quienes sostenían que en algunos centros estas nunca llegaron y en muchos solo se les había repartido un ejemplar. ${ }^{94}$

El proyecto no había cumplido con las expectativas, desde 1985 se había iniciado la elaboración. Una vez que fueron reformuladas a raíz de la impugnación de la CECOR, para 1993 entraron en funcionamiento en el sistema educativo costarricense, no obstante, su rango de aplicación fue muy limitado y rápidamente quedaron rezagadas. La polarización que provocó la creación, discusión y ejecución de las guías evidenció que, en los diversos esfuerzos que buscaban construir y regular la normalidad sexual de los y las estudiantes, no había un consenso. La versión de normalidad sexual que se quería colocar como hegemónica tuvo que ceder ante las presiones de la Iglesia.

\section{Nota sobre el autor}

José Daniel Jiménez Bolaños es Máster en Historia por la Universidad de Costa Rica y actualmente se encuentra en el programa del Doctorado en Estudios de la Sociedad y la Cultura de dicha Universidad. Es docente en la Escuela de Historia, investigador en el Centro de Investigaciones en Identidad y Cultura Latinoamericana (CIICLA) y director de la Revista

\footnotetext{
${ }^{91}$ Solís, «Papi, hablemos de sexo», 1B-2B.

92 Ivannia Varela Quirós, «Alarmante precocidad sexual», La República (San José), 12 junio de 1998, 5.

93 «Educación sexual en pañales», La República (San José), 10 junio de 1999, 10.

${ }_{94}$ Mercedes Agüero Rojas, "Guías sexuales, mucha bulla y pocos hechos», Al Día (San José), 20 octubre de 1997, 3.
} 
Reflexiones, todas de la Universidad de Costa Rica. Entre sus publicaciones más recientes se encuentran: «Los métodos de la educación sexual en Costa Rica. Normalidad sexual, Guerra Fría e institucionalización (1968-1970)» en la Revista de Historia (2021) y "La construcción de la normalidad sexual en Costa Rica entre 1930-1950» en Diálogos (2021). Actualmente se encuentra investigando acerca de la construcción de las masculinidades en el ámbito político electoral de Costa Rica durante la segunda mitad del siglo XX.

\section{REFERENCIAS}

Adams, Mary Louise. The Trouble with Normal: Postwar Youth and the Making of Heterosexuality. Toronto: University of Toronto Press, 2012.

Bonal, Xavier. "Globalización y política educativa: un análisis crítico de la agenda del Banco Mundial para América Latina». Revista Mexicana de Sociología, vol. 64, n. 3 (2002): 3-35.

Carnoy, Martin y Carlos Torres. Educational change and structural adjustment: a case study of Costa Rica. Paris: UNESCO, 1992.

Díaz, David. Reforma sin alianza, discursos transformados, interés electoral, triunfos dudosos. San José: Editorial UCR, 2010.

Foucault, Michel. El orden del discurso. Barcelona: Tusquets, 1999.

Foucault, Michel. Historia de la sexualidad vol. 1: La Voluntad del saber. Buenos Aires: Siglo XXI, 2003.

Fuentes Belgrave, Laura. "Politización evangélica en Costa Rica en torno a la agenda "provida": ¿Obra y gracia del Espíritu Santo?». Revista Rupturas, vol. 9, n. ${ }^{\circ} 1$ (2019): 85-106. https://doi.org/10.22458/rr.v9i1.2230

Guasch, Óscar. La crisis de la heterosexualidad. Barcelona: Laertes, 2000.

Katz, Jonathan. La invención de la heterosexualidad. México: Editorial Me cayó el veinte, 2012.

Molina, Iván. La educación en Costa Rica de la época colonial al presente. San José: EDUPUC, 2016.

Núñez Rivas, Hilda y Ana Rojas Chavarría. «Revisión conceptual y comportamiento del embarazo en la adolescencia en Costa Rica, con énfasis en comunidades urbanas pobres». Revista Costarricense de Salud Pública, vol. 17 , n. 13 (1998), 1-4.

Peña Bonilla, María. «Afectividad y sexualidad integral: ¿la manzana de la discordia para el sistema educativo?» Universidad de Costa Rica. https:/www.ucr.ac.cr/ noticias/2018/03/25/afectividad-y-sexualidad-integral-la-manzana-de-la-discordia-para-el-sistema-educativo.html (consultado el 18 de enero, 2021). 
Robinson, Warren y John Ross. The global family planning revolution. 3 decades of population policies and programs. Washington: The International Bank for Reconstruction and Development, 2007.

Weeks, Jeffrey. Sexuality. London: Routledge, 2003.

Zimmerman, Jonathan. A global history of sex education. New Jersey: Princeton University Press, 2015. 